\title{
High expression of atonal homolog 8 predicts a poor clinical outcome in patients with colorectal cancer and contributes to tumor progression
}

\author{
MENGSI YE ${ }^{1 *}$, YUN HE $^{1 *}$, HAO LIN ${ }^{1}$, SHOUXING YANG ${ }^{1}$, YUHUI ZHOU ${ }^{1}$, LINGLING ZHOU ${ }^{2}$, \\ JINWEI ZHONG ${ }^{1}$, GUANGRONG LU ${ }^{1}$, JIHANG ZHENG ${ }^{3}$, ZHAN-XIONG XUE ${ }^{1}$ and ZHEN-ZHAI CAI ${ }^{1}$ \\ Departments of ${ }^{1}$ Gastroenterology, ${ }^{2}$ Pathology and ${ }^{3}$ General Surgery, The Second Affiliated Hospital and \\ Yuying Children's Hospital of Wenzhou Medical University, Lucheng, Wenzhou, Zhejiang 325000, P.R. China
}

Received August 22, 2016; Accepted November 2, 2016

DOI: $10.3892 /$ or.2017.5554

\begin{abstract}
Hitherto, it has been identified that numerous basic-helix-loop-helix (bHLH) transcription factors play vital roles in tumor initiation and progression. Atonal homolog 8 (ATOH8) is a member of the bHLH family of transcription factors, which participates in embryogenesis and the development of various tissues. Several studies have demonstrated that ATOH8 is involved in the progression of malignancies; however, the effects of ATOH8 in colorectal cancer (CRC) remain unknown. The aim of the present study was to explore the expression and function of ATOH8 in CRC. The present study included 106 paired CRCs and peritumoral samples. The expression of ATOH8 was evaluated by immunohistochemistry, and the results were compared with the clinical outcomes of the patients. Furthermore, cell proliferation, cell cycle distribution, wound healing and cytotoxicity assays were performed in colon cancer cell line SW620. Immunohistochemical analyses revealed that the expression of ATOH8 in CRC tissues was significantly increased compared with the peritumoral tissues, and that the high expression of ATOH8 was associated with a high serum carcinoembryonic antigen (CEA) level and a worse overall survival. In vitro assays revealed that ATOH8 knockdown in colon cancer cells inhibited cell proliferation, induced cell cycle arrest at the $\mathrm{S}$ phase, and increased the percentage of
\end{abstract}

Correspondence to: Dr Zhen-Zhai Cai or Dr Zhan-Xiong Xue, Department of Gastroenterology, The Second Affiliated Hospital and Yuying Children's Hospital of Wenzhou Medical University, 109 Western Xueyuan Road, Lucheng, Wenzhou, Zhejiang 325000, P.R. China

E-mail:czz77@sina.com

E-mail: xuezhanxiong2016@163.com

*Contributed equally

Key words: colorectal cancer, atonal homolog 8, basic-helix-loophelix, proliferation, prognosis apoptotic cells and sensitivity to 5-fluorouracil (5-FU). The present study suggests that ATOH8 promotes the progression of CRC and may potentially serve as a novel prognostic predictor and potential therapeutic target in CRC.

\section{Introduction}

Colorectal cancer (CRC) is one of the most prevalent gastrointestinal tumors and has a high mortality rate (1). It is the third leading cause of cancer-related deaths in the US (2), and the number of CRC-related deaths in China was $\sim 159,300$ in 2012 (3). Although, the mortality rate has declined in recent decades due to the improvement of diagnosis and treatment methods, it still remains high since present therapies are limited in treating advanced CRC.

It is known that a majority of human malignancies are associated with mutations of the cancer genome. Either oncogene activation or tumor-suppressor gene inactivation leads to the initiation or acceleration, respectively, of the malignancy (4). With the improvement of array techniques, more and more genes have been reported to be involved with various types of carcinomas. Among these genes, atonal homolog 8 (ATOH8) has been identified as a new cancer-related gene. ATOH8 is a transcription factor from the basic-helix-loop-helix (bHLH) family. It is comprised of 321 characteristic amino acids with a bHLH domain and has been reported to participate in embryogenesis (5) and the development of various tissues such as the nervous system (6), pancreas (7), kidney (8), endothelial cells (9), and retina and muscle tissues $(10,11)$. Recently, it was reported that the ATOH8 gene copy number is altered in multiple malignant disorders. For instance, in hepatocellular carcinoma (HCC) (12), nasopharyngeal carcinoma (NPC) (13) and bladder cancer (14) ATOH8 mRNA expression was downregulated. However, in glioblastoma multiforme (15), prostate carcinoma (16) and breast cancer (17), the expression of ATOH8 was upregulated and considered to be a candidate oncogene.

The significance of ATOH8 alterations in CRC remains unknown. In the present study, we used immunohistochemistry to investigate the expression of ATOH8 in CRC and its correlation with clinicopathological features. We also 
conducted functional assays in vitro to explore the function of this gene in CRC.

\section{Materials and methods}

Patients and tissues. The present study was approved by the Ethics Committee of the Second Affiliated Hospital of Wenzhou Medical University, and informed consent was provided by each patient. Formalin-fixed, paraffin-embedded samples from 106 paired colon cancer tissues and peritumoral tissues were obtained from the Department of Pathology of the Second Affiliated Hospital of Wenzhou Medical University between 2010-2013. No patients received neoadjuvant chemotherapy before surgery. The histological grade was determined based on World Health Organization classification criteria by two independent pathologists. In addition, the clinical stage was determined according to Dukes' classification system. The study consisted of 68 males and 38 females ranging in age from 24 to 91 years old $(61.86 \pm 14.6)$. The clinicopathological features of the patients are summarized in Table I. The serum carcinoembryonic antigen (CEA) level was assessed by means of radioimmunoassay (RIA) (CEA-RIA kit; CIS Biointernational, China).

Follow-up. Patients were followed up with physical examination and assessment of their serum CEA level every 3-6 months in the first two years regularly. Abdominal computed tomography and colonoscopy were conducted every 6-12 months for surveillance of recurrence. In addition, we called upon the patients every three months to assess their level of discomfort. The overall survival (OS) time was defined as the time from surgery to death (the final event defined as death caused by the tumor or from complications) or the time from surgery to the last follow-up of patients. The median follow-up was 49.4 months (ranging from 1-71 months).

Immunohistochemistry and staining evaluation. Tissue sections (4- $\mu \mathrm{m}$ thick) from paraffin-embedded colon cancer and corresponding peritumoral samples were placed onto slides coated with polylysine. ATOH8 expression in colon cancer samples was examined using immunohistochemistry. In brief, sections were immersed in xylene for $10 \mathrm{~min} 3$ times, and subsequently immersed in $100 \%$ ethanol for 5 min twice for deparaffination, and then submerged in 95, 85 and $75 \%$ ethanol for $5 \mathrm{~min} /$ concentration at room temperature for rehydration. For antigen retrieval, the slides were immersed in $10 \mathrm{mM}$ citrate buffer ( $\mathrm{pH} \mathrm{6.0)}$, and heated to $120^{\circ} \mathrm{C}$ and at a pressure of $103 \mathrm{kPa}$ for $2 \mathrm{~min}$. Then, the slides were immersed in $3 \%$ hydrogen peroxide ( $20 \mathrm{~min}$, room temperature) to block endogenous peroxidase activity and $10 \%$ normal goat serum (30 min, room temperature) to block non-specific binding. The slides were then washed with phosphate-buffered saline (PBS) and treated with primary polyclonal rabbit ATOH8 antibody (ab106377; diluted 1:100; Abcam, Cambridge, MA, USA) at $4^{\circ} \mathrm{C}$ in a humidified incubator overnight. Then, the slides were washed with PBS and incubated with a secondary antibody (goat anti-rabbit IgG; Zhongshan Bio Co., Ltd., Beijing, China) for $18 \mathrm{~min}$ followed by streptavidin-peroxidase conjugate for $22 \mathrm{~min}$ at room temperature. Subsequently, the samples were stained with a 3,5-diaminobenzidine (DAB)
Table I. Relationship between ATOH8 expression and clinicopathological features.

\begin{tabular}{|c|c|c|c|c|}
\hline \multirow{2}{*}{$\begin{array}{l}\text { Clinicopathological } \\
\text { features }\end{array}$} & \multirow[b]{2}{*}{ Parameter } & \multicolumn{2}{|c|}{ ATOH8 } & \multirow[b]{2}{*}{ P-value } \\
\hline & & Low & High & \\
\hline \multirow[t]{2}{*}{ Age (years) } & $\geq 60$ & 22 & 26 & 0.918 \\
\hline & $<60$ & 26 & 32 & \\
\hline \multirow[t]{2}{*}{ Sex } & Male & 27 & 41 & 0.123 \\
\hline & Female & 21 & 17 & \\
\hline \multirow[t]{2}{*}{ Tumor site } & Right colon & 18 & 29 & 0.197 \\
\hline & Left colon & 30 & 29 & \\
\hline \multirow[t]{2}{*}{ Size } & $>5 \mathrm{~cm}$ & 17 & 26 & 0.433 \\
\hline & $\leq 5 \mathrm{~cm}$ & 30 & 33 & \\
\hline \multirow[t]{2}{*}{$\begin{array}{l}\text { Histological } \\
\text { grade }\end{array}$} & $\begin{array}{l}\text { High to } \\
\text { moderate }\end{array}$ & 24 & 33 & 0.478 \\
\hline & Low & 24 & 25 & \\
\hline \multirow[t]{2}{*}{ Dukes' stage } & A-B & 28 & 28 & 0.333 \\
\hline & C-D & 20 & 30 & \\
\hline \multirow[t]{2}{*}{ Serum CEA (ng/ml) } & $<5$ & 38 & 34 & 0.036 \\
\hline & $\geq 5$ & 10 & 24 & \\
\hline $\begin{array}{l}\text { Mean survival time } \\
\text { (95\% CI) (months) }\end{array}$ & & $\begin{array}{c}61.8 \\
(56.7-67.0)\end{array}$ & $\begin{array}{c}51.5 \\
(45.2-57.7)\end{array}$ & 0.022 \\
\hline
\end{tabular}

CEA, carcinoembryonic antigen; CI, confidence interval.

substrate kit (Zhongshan Bio Co., Ltd.) and haematoxylin for counterstaining. All of the samples were evaluated by two independent pathologists who were blinded to the clinical data of the patients. The inconsistent cases were reassessed on a double-headed microscope. Immunohistochemical evaluation of ATOH8 protein expression was performed as previously described, including staining intensity and extent (18). The intensity score was as follows: 0 , negative; 1 , weak; 2 , moderate; and 3, strong. The extent of positivity score was quantified from the percentage of positive tumor cells: $0,<5 \%$; $1, \geq 5-25 \% ; 2, \geq 26-50 \% ; 3, \geq 51-75 \%$; and $4, \geq 76 \%$. The final score was determined by multiplying the intensity and extent scores, which ranged between 0 and 12 ; scores $\leq 4$ indicated low expression.

Quantitative RT-PCR. Total RNA was extracted from cultured cells with TRIzol reagent (Superfec Bio Co., Ltd., Shanghai, China) according to the manufacturer's instructions. Then, an M-MLV reverse transcriptase kit (Promega Bio Co., Ltd., Beijing, China) was used for reverse transcription. To determine the transcripts of the target genes, quantitative real-time polymerase chain reaction (qRT-PCR) was conducted with a SYBR Premix Ex Taq (RibBio Co., Ltd., Guangzhou, China) on an Agilent MX3000p Bioanalyzer (Applied Biosystems Co., Ltd., CA, USA). qRT-PCR conditions were as follows: $95^{\circ} \mathrm{C}$ for $30 \mathrm{sec} ; 45$ cycles of $95^{\circ} \mathrm{C}$ for $5 \mathrm{sec}, 60^{\circ} \mathrm{C}$ for $30 \mathrm{sec}$, and $95^{\circ} \mathrm{C}$ for $15 \mathrm{sec} ; 55^{\circ} \mathrm{C}$ for $30 \mathrm{sec}$; and $95^{\circ} \mathrm{C}$ for $15 \mathrm{sec}$. Three independent experiments were run in triplicate. The relative amount of ATOH8 mRNA was normalized to the 
control GAPDH. The primer sequences were as follows: ATOH8, 5'-TGGGCAGAAGCTGTCCAAACT-3' and GTGG TCGGCACTGTAGTCAAG, and GAPDH, 5'-TGACTTCAA CAGCGACACCCA-3' and 5'-CACCCTGTTGCTGTAGCC AAA-3'.

Western blotting. CRC cells were harvested and washed with cold PBS and lysed with radio-immunoprecipitation assay (RIPA) lysis buffer (Beyotime Bio Co., Ltd., Haimen, China). Cell debris was centrifuged and total protein in the supernatants was assessed with a BCA protein assay kit (Beyotime). Amounts equal to $50 \mu \mathrm{g}$ of protein were separated on a $10 \%$ SDS-PAGE gel, then transferred to a polyvinylidene fluoride (PVDF) membrane (Beyotime) and incubated with the ATOH8 (ab106377; diluted 1:800) and GAPDH (ab8245; diluted 1:5,000) (both from Abcam) primary antibodies at $4^{\circ} \mathrm{C}$ overnight. Then, the membrane was incubated with the HRP-labelled goat antirabbit IgG secondary antibody (diluted 1:1,000; Beyotime) at room temperature for $2 \mathrm{~h}$. Proteins were visualized with enhanced chemiluminescence detection reagents (Applygen Technologies, Beijing, China). The relative amount of ATOH8 protein was normalized to the control GAPDH.

Cell culture and lentiviral infection. Four types of human colon carcinoma cell lines (HCT116, SW620, RKO and LoVo) were purchased from the Shanghai Cell Bank of the Chinese Academy of Sciences. All of the cancer cell lines were cultured in high-glucose Dulbecco's modified Eagle's medium (DMEM) with $10 \%$ foetal bovine serum (FBS) (Atlanta Biologicals, Lawrenceville, GA, USA) at $37^{\circ} \mathrm{C}$ in $5 \% \mathrm{CO}_{2}$ moist incubator. All experiments were performed on exponentially growing cells. Based on the sequence of ATOH8 (NM_032827.6), we designed three different small interfering RNAs (siRNAs) (siRNA\#1, 5'-CGTCAATTTCACACGTAAT-3'; siRNA\#2, 5'-ACGGCCTTAAGAAGCTCAA-3'; and siRNA\#3, 5'-TGA GGATCGCCTGTAACTA-3'), and a negative control (NC) siRNA (5'-TTCTCCGAACGTGTCACGT-3'). The ATOH8-specific and negative control lentiviral packaging plasmids were purchased from GeneChem Biotechnologies, Co., Ltd. (Shanghai, China). The lentiviral vectors which expressed green fluorescent protein (GFP) were transfected into the CRC cell lines using the Lipofectamine 2000 reagent (Invitrogen, Carlsbad, CA, USA) in accordance with the manufacturer's instructions. A fluorescence microscope (Olympus-BX53; Olympus, Tokyo, Japan) confirmed whether the target cells were transfected with the lentiviral vectors.

Cell proliferation and cytotoxicity assay. Cells were seeded into a 96-well plate at a density of $2.0 \times 10^{4}$ cells $/ \mathrm{ml}$ and incubated in a humidified environment of $5 \% \mathrm{CO}_{2}$ at $37^{\circ} \mathrm{C}$. On day $1-5$, the medium was removed and $20 \mu 1$ of $5 \mathrm{mg} / \mathrm{ml}$ MTT (Genview, Tallahassee, FL, USA) was added to each well, respectively. Four hours later, the MTT reagent was removed and replaced with $100 \mu 1$ of dimethyl sulfoxide (DMSO) to stop the reaction, and then the plates were agitated for $5 \mathrm{~min}$. Subsequently, the absorbance of the samples was assessed at $490 \mathrm{~nm}$ with a microplate reader.

Cell chemosensitivity to 5-fluorouracil (5-FU) was determined by MTT assay. Approximately $2 \times 10^{4}$ cells/well were seeded in 96-well plates, allowed to attach overnight, and exposed to different concentrations of 5-FU from 1 to $150 \mu \mathrm{g} / \mathrm{ml}$ for $24 \mathrm{~h}$. Then, an MTT assay was performed as aforementioned.

Cell apoptosis analysis. Following five days of transfection, apoptosis was assessed by the Annexin V-APC apoptosis detection kit (eBioscience, San Diego, CA, USA) according to the manufacturer's instructions. At first, cells seeded in 6-well dishes were trypsinized, collected and incubated in $80 \%$ ethanol at $4^{\circ} \mathrm{C}$ overnight. Then, the cells were stained with Annexin V-APC at room temperature in the dark. The apoptosis rate was analysed by flow cytometry (BD Biosciences, San Diego, CA, USA) within $1 \mathrm{~h}$. Cells positive for Annexin V-APC were considered to be apoptotic cells.

Cell cycle analysis. SW620 cells $\left(2 \times 10^{6}\right)$ were seeded in 6-cm dishes and trypsinized, collected and washed with PBS twice. Prior to staining with propidium iodide (PI), the cells were incubated in $80 \%$ ethanol at $4^{\circ} \mathrm{C}$ overnight. Subsequently, the stained cells were assessed by flow cytometry. The fraction of cells in the G0/G1, S and G2/M phases was analysed using CellQuest software programs.

Cell wound-healing assay. In the cell motility assay, stably-transfected ATOH8-siRNA cells were cultured until cell confluence reached $90 \%$. Then, a $200 \mu \mathrm{l}$ pipette tip was used to draw a wound at the bottom of each well. The cells were washed with PBS, and then further cultured in $0.5 \%$ FBS at $37^{\circ} \mathrm{C}$ with $5 \% \mathrm{CO}_{2}$. Wound closure was observed after 8 and $24 \mathrm{~h}$. We used the relative migration rate to evaluate the motility of cells. Relative migration rate $=$ distance of migration/the width at $0 \mathrm{~h}(\%)$. Distance of migration $=$ the width at $0 \mathrm{~h}$ - the width at time (mm).

Statistical analysis. All statistical analyses were performed with SPSS 22.0 (SPSS, Inc., Chicago, IL, USA). For numerical variables, the data was expressed as the means \pm SEM. The significance of the differences between the values was determined by Student's t-test. The correlation between ATOH8 and the clinicopathological features was assessed by Chi-squared and Fisher's exact tests. The OS time was calculated by the Kaplan-Meier method, and the differences were analysed by the log-rank test. A P-value $<0.05$ was considered to indicate a statistically significant result.

\section{Results}

Expression of ATOH8 in colon cancer patients and its correlation with clinicopathological characteristics. To explore the role of ATOH8 in colon cancer, we surveyed the expression of ATOH8 in 106 paired colon cancer samples using immunohistochemistry. Our results indicated that ATOH8 displayed either diffuse or localized patterns in the cytoplasm of both cancer and peritumoral tissues (Fig. 1A and B). According to our evaluation criteria, the ATOH8 protein was highly expressed in $58 / 106$ (54.7\%) of colon cancer samples but only $7 / 106$ (6.6\%) of peritumoral samples $(\mathrm{P}=0.000)$. Based on the level of ATOH8 expression in the tumor-cell cytoplasm, patients were divided into an ATOH8 low expression group (including negative and low expression) and a high expression group (high expression; 

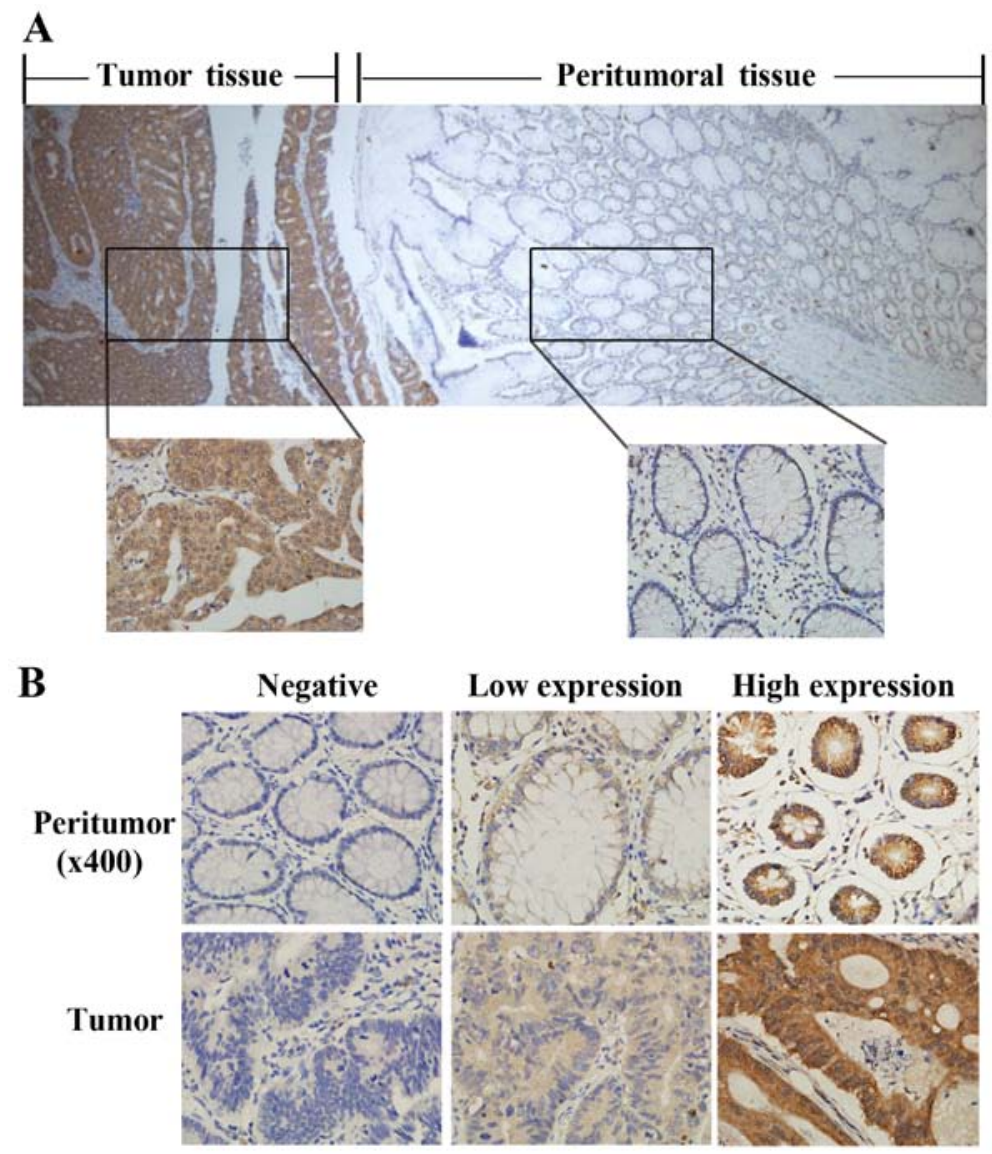

Figure 1. Immunohistochemical staining of ATOH8 in colorectal carcinoma and peritumoral tissues. (A) ATOH8 was diffusely expressed in the cytoplasm of tumor cells, but sporadically expressed or negative in adjacent non-neoplastic colon tissues. (B) Representative photomicrographs of negative, low and high ATOH8 expression in both tumor and peritumoral tissues (magnification, x400). ATOH8, atonal homolog 8.
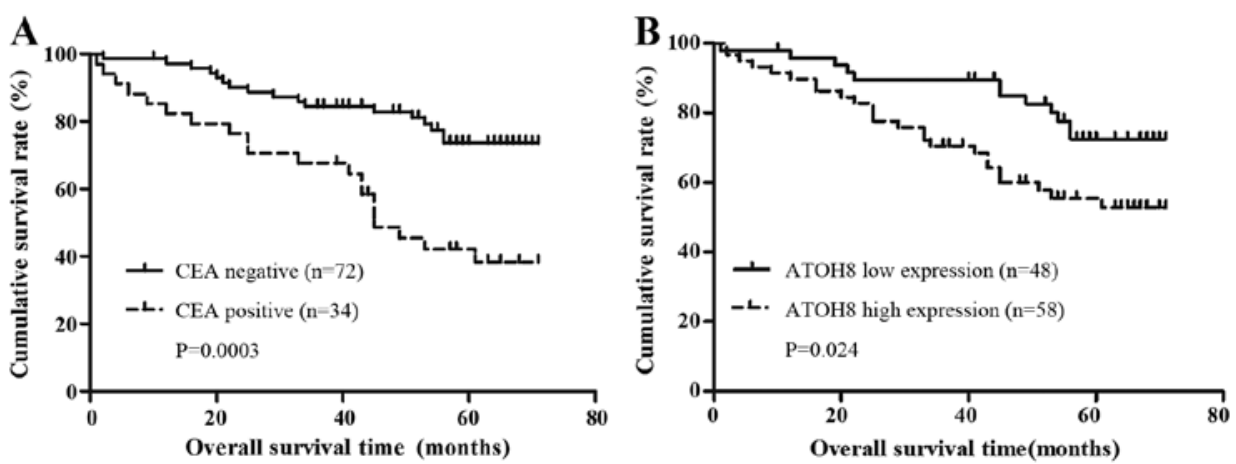

Figure 2. Positive CEA and ATOH8 expression is related to worse prognosis of CRC patients. (A) The overall survival time of the CEA-positive patients was significantly shorter than the CEA-negative patients $(\mathrm{P}=0.0003)$. (B) The Kaplan-Meier analysis for overall survival revealed that $\mathrm{OS}$ was decreased in the ATOH8 high expression group compared to the low expression group $(\mathrm{P}=0.024)$. CEA, carcinoembryonic antigen; ATOH8, atonal homolog 8; CRC, colorectal cancer.

Fig. 1B). We explored the relationship between the expression level of ATOH8 and clinicopathological features. High expression of ATOH8 was significantly associated with a high serum CEA level, but there was no connection between the expression level of ATOH8 with age, gender, tumor location, tumor size, histological grade or Dukes' stage (Table I). We also compared the serum CEA level with the prognosis of CRC patients by Kaplan-Meier survival analysis. It suggested that the patients with a high level of pre-surgery CEA had a shorter OS time (95\% CI, 37.3-54.1 months vs. 95\% CI, 56.9-65.6 months,
$\mathrm{P}=0.0003$; Fig. 2A). Moreover, when comparing the expression of ATOH8 with OS, the high expression group was observed to have statistically worse OS (95\% CI, 45.2-57.7 months vs. 95\% CI, 56.7-67.0 months; $\mathrm{P}=0.024$, Fig. 2B).

Lentivirus-mediated ATOH8 knockdown in CRC cells. The expression of ATOH8 mRNA was analysed in the four colon cancer cell lines HCT116, SW620, LoVo and RKO with qRT-PCR. The relative expression ratio of ATOH8 mRNA in the four cancer cell lines was normalized to LoVo cells. 
A

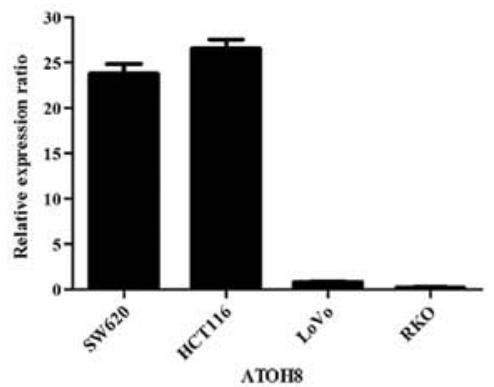

B

ATOH8

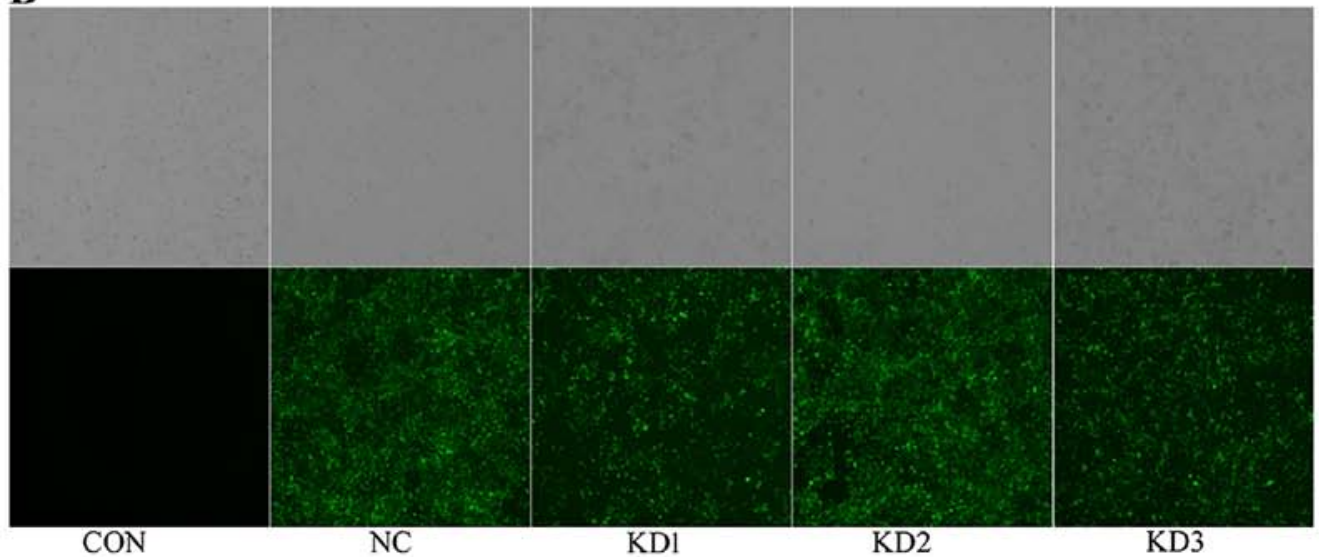

CON

NC
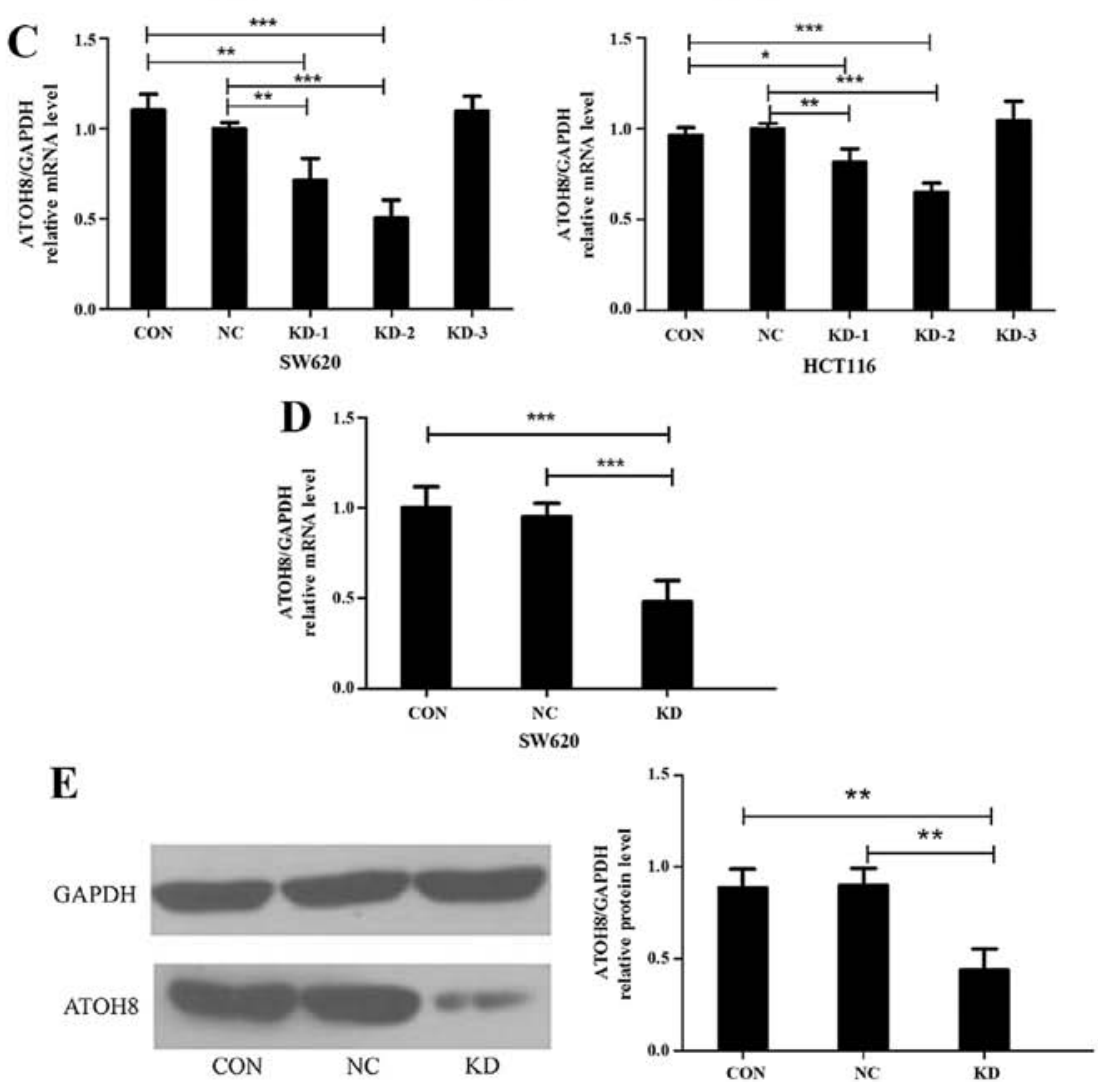

Figure 3. Lentiviral-mediated ATOH8 knockdown in CRC cells. (A) Relative expression ratio of ATOH8 in SW620, HCT116, LoVo and RKO colon cancer cell lines as normalized to LoVo cells. The expression levels of ATOH8 in SW620 and HCT116 cells were relatively high. (B) The transfection of different GFP-lentiviral siRNA vectors (NC, KD1, KD2 and KD3) in SW620 cells was evaluated by fluorescence microscopy. (C) The knockdown efficiency of each lentiviral siRNA vector in SW620 and HCT116 cells was confirmed by qRT-PCR, and it revealed that ATOH8 expression was effectively downregulated by both KD1 and KD2 compared with CON and NC. The expression of ATOH8 in the CON, NC and KD groups was confirmed by (D) qRT-PCR and (E) western blotting. CON, non-transfected control group; NC, negative control group; KD, ATOH8-siRNA transfection group; ${ }^{*} \mathrm{P}<0.05,{ }^{* *} \mathrm{P}<0.01,{ }^{* * *} \mathrm{P}<0.001$. ATOH8, atonal homolog 8; CRC, colorectal cancer; GFP, green fluorescent protein.

The relative expression levels of ATOH8 mRNA in the four cancer cell lines were $26.58 \pm 1.75,23.85 \pm 1.74,0.81 \pm 0.19$ and
$0.23 \pm 0.06$ in the HCT116, SW620, LoVo and RKO cells, respectively (Fig. 3A). Expression in the SW620 and HCT116 


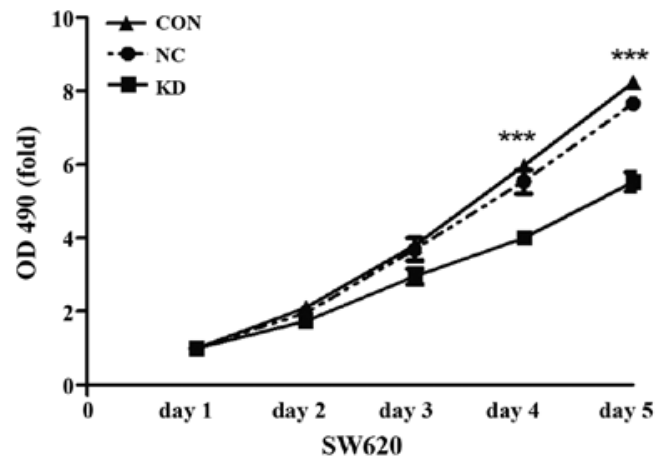

Figure 4. Knockdown of ATOH8 suppresses CRC cell proliferation. The MTT assay indicated that ATOH 8 knockdown inhibited the proliferative ability of SW620 cells on day 4 and $5 ;{ }^{* * *} \mathrm{P}<0.001$. CON, non-transfected control group; NC, negative control group; KD, ATOH8-siRNA transfection group; ATOH8, atonal homolog 8; CRC, colorectal cancer.

cells was relatively high, so we chose these two cell lines in the following experiment. Then, we designed a negative control siRNA (NC) and three different ATOH8 siRNAs (KDs) (siRNA\#1, siRNA\#2, siRNA\#2) and cloned them into GFP-lentiviral vectors. The groups transfected with $\mathrm{NC}$ and KDs were visible in the dark under fluorescence microscopy, while the non-transfected control group (CON) was invisible, which means the lentiviral vectors were successfully transfected into the SW620 (Fig. 3B) and HCT116 cells (data not shown). Then, we screened the expression of ATOH8 mRNA in SW620 and HCT116 cells using qRT-PCR to ascertain the knockdown efficiency. The results suggested that ATOH8 expression was effectively downregulated by siRNA\#1 (KD1, decreased to $71.6 \pm 11.9 \%$ and $81.6 \pm 7.3 \% ; \mathrm{P}<0.05$ ) and siRNA\#2 (KD2, decreased to $50.3 \pm 10.0 \%$ and $65.0 \pm 5.0 \%$; $\mathrm{P}<0.001)$ in the SW620 and HCT116 cells, respectively, compared to the controls. However, siRNA\#3 (KD3) did not decrease ATOH8 expression in either the SW620 (109.7 $\pm 8.2 \%)$ or HCT116 (104.4 $\pm 7.3 \%$ ) cells (Fig. 3C). As the knockdown efficiency of KD2 in SW620 cells was the highest, we chose the siRNA\#2-SW620 cells in the following experiments. Before additional in vitro experiments, the expression of ATOH8 of the CON, NC and KD groups was confirmed by qRT-PCR (Fig. 3D; $\mathrm{P}<0.001$ ) and western blotting (Fig. 3E; $\mathrm{P}<0.01$ ).

Knockdown of ATOH8 suppresses CRC cell proliferation. We performed an MTT assay to investigate the effect of ATOH8 knockdown on the proliferation ability of SW620 cells. As shown in Fig. 4, the proliferation of the KD group on day 4 and 5 was markedly decreased compared with the $\mathrm{CON}$ and $\mathrm{NC}$ group $(\mathrm{P}<0.001)$.

Knockdown of ATOH8 increases cell apoptosis. Following five days of transfection, the confluence of the cells reached $\sim 85 \%$ in the CON, $\mathrm{NC}$ and $\mathrm{KD}$ groups, and then we performed Annexin V-APC single staining assay (Fig. 5A and B). As
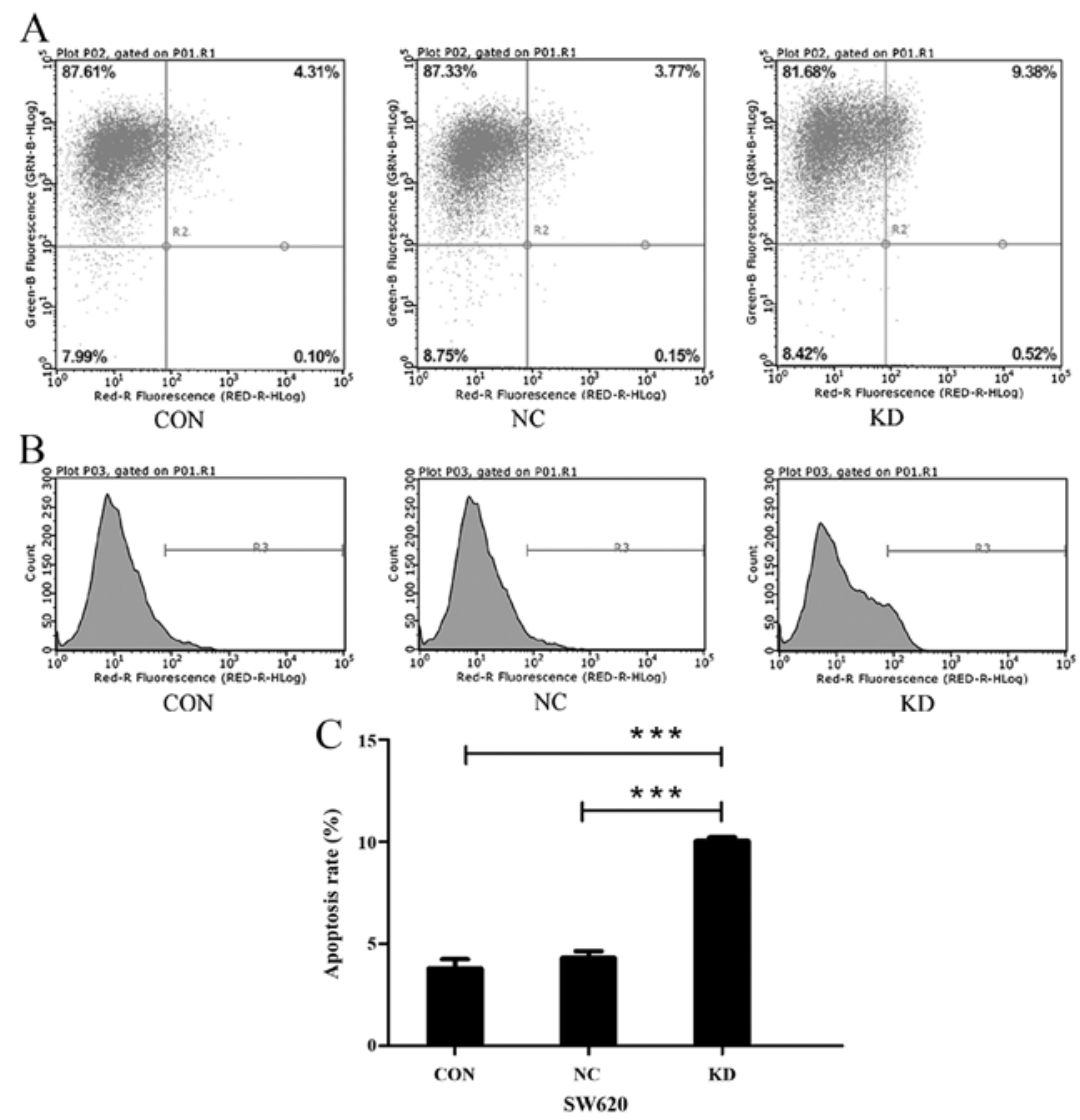

Figure 5. Knockdown of ATOH8 increases cell apoptosis. (A) Annexin V-APC was set as the horizontal axis. Cells positive for Annexin V-APC were considered to be apoptotic cells. (B and C) The Annexin V-APC single-staining assay revealed that the percentage of apoptotic SW620 cells in the KD group $(10.04 \pm 0.1778 \%)$ was significantly increased compared to the $\mathrm{NC}(4.3 \pm 0.342 \%)$ and CON $(3.8 \pm 0.445 \%)$ groups; ${ }^{* * *} \mathrm{P}<0.001$. CON, non-transfected control group; NC, negative control group; KD, ATOH8-siRNA transfection group; ATOH8, atonal homolog 8. 


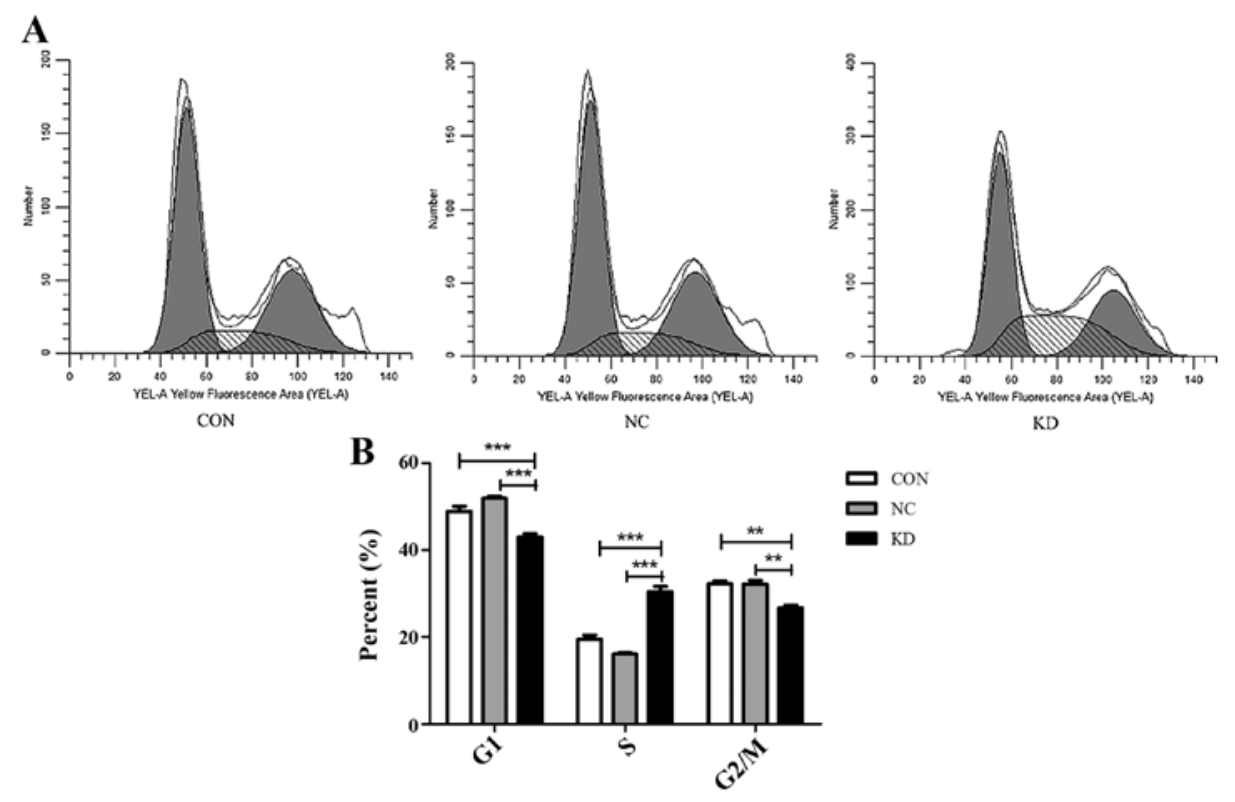

Figure 6. (A) Flow cytometric analysis of the cell cycle. (B) The percentage of cells in the G1 and G2/M phase was significantly decreased in the knockdown ATOH8 cells while the percentage in the $\mathrm{S}$ phase was increased; ${ }^{* *} \mathrm{P}<0.01,{ }^{* * *} \mathrm{P}<0.001$. CON, non-transfected control group; NC, negative control group; KD, ATOH8-siRNA transfection group; ATOH8, atonal homolog 8.
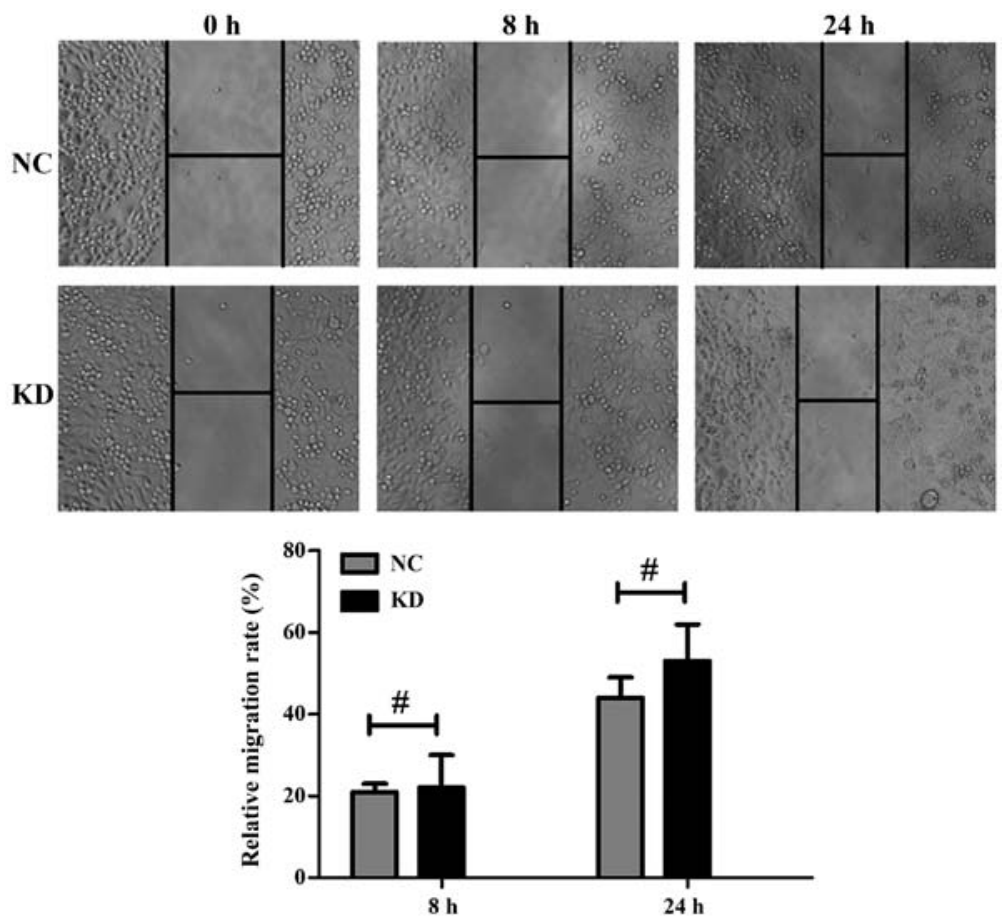

Figure 7. The wound healing assay indicates that ATOH8 knockdown has no effect on cell migration in SW620 cells at 8 or $24 \mathrm{~h} ;{ }^{*} \mathrm{P}>0.05$. NC, negative control group; KD, ATOH8-siRNA transfection group; ATOH8, atonal homolog 8.

shown in Fig. 5C, the percentage of apoptotic cells in the KD group $(10.04 \pm 0.178 \%)$ was significantly increased compared to the CON group $(3.8 \pm 0.445 \% ; \mathrm{P}<0.001)$ and $\mathrm{NC}$ group $(4.3 \pm 0.342 \% ; \mathrm{P}<0.001)$.

Knockdown of ATOH8 arrests the cell cycle in the S phase. To investigate the role of ATOH8 knockdown in cell cycle progression, flow cytometry was performed, which revealed that ATOH8 knockdown arrested the cell cycle in SW620 cells. As shown in Fig. 6, the percentage of the G1 phase cells (CON vs.
$\mathrm{NC}$ vs. KD, 48.87 vs. 51.87 vs. $42.93 \%$, respectively; $\mathrm{P}<0.001)$ and the $\mathrm{G} 2 / \mathrm{M}$ phase cells (32.15 vs. 32.12 vs. $26.69 \%$; $\mathrm{P}<0.01$ ) was significantly decreased in the ATOH8-knockdown group while the percentage of cells in the $S$ phase (19.49 vs. 16.02 vs. $30.38 \%$; $\mathrm{P}<0.001)$ was increased. Collectively, these data demonstrated that ATOH8 knockdown led to cell cycle arrest during the $\mathrm{S}$ phase.

ATOH8 knockdown has no effect on the cell motility of CRC cells. The wound healing assay was used to determine the 


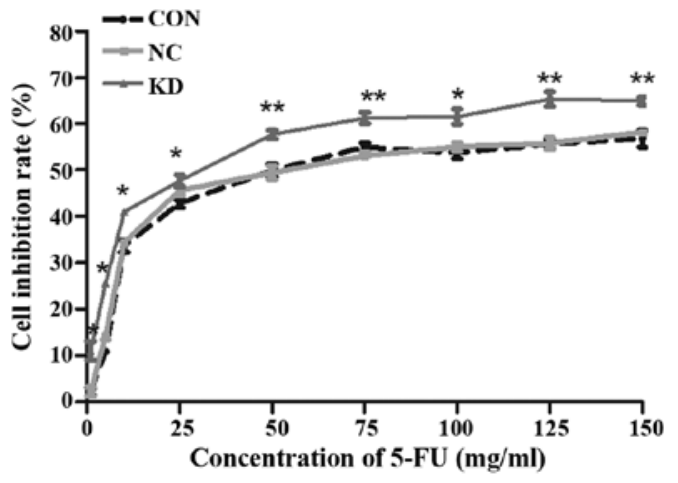

Figure 8. ATOH8 knockdown increases the sensitivity of CRC cells to 5-FU. The inhibitory rate of 5-FU was dose-dependent in the CON, NC and KD groups. The inhibitory rate of the KD group was higher than that of the $\mathrm{CON}$ and $\mathrm{NC}$ groups at the same drug concentration; ${ }^{*} \mathrm{P}<0.05,{ }^{* * *} \mathrm{P}<0.01$. NC, negative control group; KD, ATOH8-siRNA transfection group. ATOH8, atonal homolog 8; CRC, colorectal cancer; 5-FU; 5-fluorouracil.

effects of ATOH8 downregulation on cell motility. As shown in Fig. 7, the wound healing assay indicated ATOH8 knockdown had no effect on the cell migration rate of SW620 cells. The relative migration rate at $8 \mathrm{~h}$ of $\mathrm{NC}$ and $\mathrm{KD}$ group was $21 \pm 2$ and $22 \pm 8 \%$, respectively, $\mathrm{P}>0.05$; and migration rate at 24 h was $44 \pm 5$ and $53 \pm 9 \%$, respectively, $\mathrm{P}>0.05$.

ATOH8 knockdown increases the sensitivity of CRC cells to 5-FU. The effect of ATOH8 knockdown on drug sensitivity was performed by evaluating the cytotoxicity of 5-FU in SW620 cells. The inhibitory effect of various concentrations of 5-FU was assessed by MTT assay following $24 \mathrm{~h}$ of treatment. The inhibitory effect of 5-FU was dose-dependent in the $\mathrm{CON}, \mathrm{NC}$ and KD groups (Fig. 8). In addition, the inhibitory rate of the KD group was increased more significantly than the $\mathrm{CON}$ and $\mathrm{NC}$ groups as the drug concentration increased, which meant that ATOH8 knockdown increased the sensitivity of SW620 to 5-FU.

\section{Discussion}

ATOH8 belongs to the atonal family and consists of 321 amino acids with a bHLH domain. It has been shown that ATOH8 has important regulatory functions during the differentiation of pancreatic precursor cells, neurons, podocytes, skeletal muscle, retina and endothelial cells $(6,8-11,19,20)$. With the improvement of array techniques, it was found that ATOH8 exhibited abnormal expression in various cancers such as prostate and breast cancer, glioblastoma multiforme and hepatocellular carcinoma $(12,13,15-17)$. Song et al (12) focused on the function of ATOH8 in hepatocellular cancer. They demonstrated that ATOH8 expression was decreased in hepatocellular cancer, and that the low expression of ATOH8 was associated with poor differentiation, high serum AFP level and worse overall survival. The same researchers constructed ATOH8 overexpression and low-expression models in HCC cell lines and demonstrated that upregulation of ATOH8 decreased tumor proliferation, invasion, and migratory abilities as well as increased the sensitivity of HCC cell lines to chemotherapy, while downregulation of ATOH8 showed the opposite effects. Moreover, Wang et al (13) designed a series of experiments in nasopharyngeal carcinoma (NPC) and demonstrated that the inhibition of ATOH8 expression promoted a malignant phenotype of NPC and that this malignant phenotype could be reversed by ATOH8 restoration. These two studies suggested that ATOH8 acted as a tumor-suppressor gene in HCC and NPC. However, a study in breast cancer (17) suggested that ATOH8 was a downstream effector of IL-6/STAT3 signaling, which increased the stemness potential of breast cancer cell lines. Thus, the function of ATOH8 in cancer is controversial. The role of ATOH8 in colorectal cancer (CRC) is currently unknown, thus, we investigated the expression of ATOH8 in $\mathrm{CRC}$ tissues and the function of this gene in vitro.

By comparing 106 biopsies of carcinoma tissues with their corresponding peritumoral tissues, immunohistochemical results demonstrated that the expression of ATOH8 was clearly increased in the carcinoma tissues. Bowden et al (21) demonstrated that in familial adenomatous polyposis adenomas, a high risk subset of CRC, ATOH8 expression was increased compared with normal colon epithelial cells, which supports our results. When we compared the expression of ATOH8 with clinicopathological features, we found that high expression of ATOH8 was associated with a high serum CEA level. There are several studies that have shown that a high serum CEA level is a poor prognosis predictor for CRC patients (22-24). Thus, we also investigated the relationship between the serum CEA level and the OS of the patients, and the result was consistent with former studies (22-24). Furthermore, our results based on the Kaplan-Meier analysis revealed that patients with high levels of ATOH8 expression had a shorter overall survival time than those with low expression. Considering all these results, we proposed that the expression of ATOH8 is a potential prognostic factor of colorectal carcinoma.

To explore the function of ATOH8 in colon cancer, we suppressed ATOH8 expression in SW620 cells through ATOH8-siRNA transfection. We successfully constructed a downregulated ATOH8 model confirmed by western blotting and qRT-PCR. The MTT assay suggested that the growth rate of CRC cells was significantly decreased in the downregulated ATOH8 group compared to the control and negative groups, which suggests that ATOH8 promotes cell proliferation in CRC cell lines. Studies have demonstrated that different types of bHLH transcriptional factors have marked similarity in many functional regions (25), and numerous bHLH transcriptional factors play important roles in tumor growth and proliferation. For example, Ascle2, a bHLH transcriptional factors (TF), promoted cellular proliferation in CRC (26), and E2A (i.e., isoforms E12 and E47) and accelerated the cellular growth rate in prostate cancer (27).

Flow cytometric analysis suggested that the proportion of cells in the G0/G1 phase increased while the percentage of cells in the $\mathrm{S}$ and $\mathrm{G} 2 / \mathrm{M}$ phase decreased, which means that ATOH8 depletion induced $\mathrm{S}$ phase arrest accompanied by a decrease in cell proliferation. Additionally, ATOH8 depletion increased the proportion of apoptotic CRC cells. There are no other studies that have been conducted concerning the effect of ATOH8 on the cell cycle and apoptosis in malignancy, thus far. However, it has been reported that some bHLH transcriptional factors, such as Hes1, through its inhibitor induced apoptosis in cancer cells by inhibiting the Notch signaling 
pathway. Currently, some Hes1 inhibitors are in clinical trials $(28,29)$.

The effect of ATOH8 on the cell motility of CRC cells was also investigated by wound healing assay. The results indicated that ATOH8 depletion did not affect the cell motility of colon cancer cells. Although, in HCC and NPC $(12,13)$, the expression of ATOH8 was associated with the invasive and migratory abilities of the cancer, in these two tumor types ATOH8 functioned as a cancer suppressor gene.

The potential therapeutic value of ATOH8 in CRC treatment was also investigated. From our results, the inhibitory rate of the ATOH8-downregulated cells was higher than the controls at different concentrations of 5-FU, which implies that downregulation of ATOH8 increases the sensitivity of colon cancer cells to chemotherapy. It also suggests that ATOH8 may be a potential therapeutic target for CRC.

The first major limitation of the present study was the fact that we did not explore the molecular mechanism of ATOH8 in CRC. Secondly, we only explored the function of ATOH8 in an in vitro cell model without in vivo experiments. Our next goal is to find the downstream effector of ATOH8 and the corresponding signaling pathways.

In summary, our data demonstrated that ATOH8 expression was upregulated in CRC patients, and that it predicted poor prognosis. The upregulation of ATOH8 may increase the malignancy potential of CRC. Finally, ATOH8 is a potential therapeutic target of CRC.

\section{Acknowledgements}

The present study received research funding from the Zhejiang Provincial Natural Science Foundation of China (LY15H160059).

\section{References}

1. Torre LA, Bray F, Siegel RL, Ferlay J, Lortet-Tieulent J and Jemal A: Global cancer statistics, 2012. CA Cancer J Clin 65: 87-108, 2015.

2. Siegel R, Desantis C and Jemal A: Colorectal cancer statistics, 2014. CA Cancer J Clin 64: 104-117, 2014.

3. Chen W, Zheng R, Zuo T, Zeng H, Zhang S and He J: National cancer incidence and mortality in China, 2012. Chin J Cancer Res 28: 1-11, 2016.

4. Weir B, Zhao X and Meyerson M: Somatic alterations in the human cancer genome. Cancer Cell 6: 433-438, 2004.

5. Wang B, Balakrishnan-Renuka A, Napirei M, Theiss C and Brand-Saberi B: Spatiotemporal expression of Math6 during mouse embryonic development. Histochem Cell Biol 143: 575-582, 2015.

6. Inoue $\mathrm{C}$, Bae SK, Takatsuka $\mathrm{K}$, Inoue $\mathrm{T}$, Bessho $\mathrm{Y}$ and Kageyama R: Math6, a bHLH gene expressed in the developing nervous system, regulates neuronal versus glial differentiation. Genes Cells 6: 977-986, 2001.

7. Lynn FC, Sanchez L, Gomis R, German MS and Gasa R: Identification of the bHLH factor Math6 as a novel component of the embryonic pancreas transcriptional network. PLoS One 3: e2430, 2008.

8. Ross MD, Martinka S, Mukherjee A, Sedor JR, Vinson C and Bruggeman LA: Math6 expression during kidney development and altered expression in a mouse model of glomerulosclerosis. Dev Dyn 235: 3102-3109, 2006.

9. Fang F, Wasserman SM, Torres-Vazquez J, Weinstein B, Cao F, Li Z, Wilson KD, Yue W, Wu JC, Xie X, et al: The role of Hath6, a newly identified shear-stress-responsive transcription factor, in endothelial cell differentiation and function. J Cell Sci 127: 1428-1440, 2014.
10. Güttsches AK, Balakrishnan-Renuka A, Kley RA, Tegenthoff M, Brand-Saberi B and Vorgerd M: ATOH8: A novel marker in human muscle fiber regeneration. Histochem Cell Biol 143: 443-452, 2015.

11. Yao J, Zhou J, Liu Q, Lu D, Wang L, Qiao X and Jia W: Atoh8, a bHLH transcription factor, is required for the development of retina and skeletal muscle in zebrafish. PLoS One 5: e10945, 2010.

12. Song Y, Pan G, Chen L, Ma S, Zeng T, Man Chan TH, Li L, Lian Q, Chow R, Cai X, et al: Loss of ATOH8 increases stem cell features of hepatocellular carcinoma cells. Gastroenterology 149: 1068-1081.e5, 2015.

13. Wang Z, Xie J, Yan M, Wang J, Wang X, Zhang J, Zhang Y, Li P, Lei X, Huang Q, et al: Downregulation of ATOH8 induced by EBV-encoded LMP1 contributes to the malignant phenotype of nasopharyngeal carcinoma. Oncotarget 7: 26765-26779, 2016.

14. Zaravinos A, Lambrou GI, Boulalas I, Delakas D and Spandidos DA: Identification of common differentially expressed genes in urinary bladder cancer. PLoS One 6: e18135, 2011.

15. Freire P, Vilela M, Deus H, Kim YW, Koul D, Colman H, Aldape KD, Bogler O, Yung WK, Coombes K, et al: Exploratory analysis of the copy number alterations in glioblastoma multiforme. PLoS One 3: e4076, 2008.

16. Hazelett DJ, Rhie SK, Gaddis M, Yan C, Lakeland DL, Coetzee SG, Henderson BE, Noushmehr H, Cozen W, Kote-Jarai Z, et al; Ellipse/GAME-ON consortium; Practical consortium: Comprehensive functional annotation of 77 prostate cancer risk loci. PLoS Genet 10: e1004102, 2014.

17. Chang A, Chen Y, Shen W, Gao R, Zhou W, Luo Y, Luo N, Stupack D and Xiang R: Abstract 1956: The basic helix-loophelix (bHLH) transcriptional factor ATOH8 promotes the stemness of breast cancer cells via Oct4 and Nanog. Cancer Res 74 (Suppl 19): Abstract nr 1956, 2014. doi:10.1158/1538-7445. AM2014-1956.

18. Chu X, Zhao P, Lv Y and Liu L: Decreased expression of TFPI-2 correlated with increased expression of CD133 in cholangiocarcinoma. Int J Clin Exp Pathol 8: 328-336, 2015.

19. Ejarque M, Mir-Coll J, Gomis R, German MS, Lynn FC and Gasa R: Generation of a conditional allele of the transcription factor atonal homolog 8 (Atoh8). PLoS One 11: e0146273, 2016.

20. Balakrishnan-Renuka A, Morosan-Puopolo G, Yusuf F, Abduelmula A, Chen J, Zoidl G, Philippi S, Dai F and Brand-Saberi B: ATOH8, a regulator of skeletal myogenesis in the hypaxial myotome of the trunk. Histochem Cell Biol 141: 289-300, 2014.

21. Bowden NA, Croft A and Scott RJ: Gene expression profiling in familial adenomatous polyposis adenomas and desmoid disease. Hered Cancer Clin Pract 5: 79-96, 2007.

22. Tampellini M, Ottone A, Alabiso I, Baratelli C, Forti L, Berruti A, Aroasio E and Scagliotti GV: The prognostic role of baseline CEA and CA 19-9 values and their time-dependent variations in advanced colorectal cancer patients submitted to first-line therapy. Tumor Biol 36: 1519-1527, 2015.

23. Yang KL, Yang SH, Liang WY, Kuo YJ, Lin JK, Lin TC, Chen WS, Jiang JK, Wang HS, Chang SC, et al: Carcinoembryonic antigen (CEA) level, CEA ratio, and treatment outcome of rectal cancer patients receiving pre-operative chemoradiation and surgery. Radiat Oncol 8: 43, 2013.

24. Chen L, Jiang B, Di J, Zhang C, Wang Z, Zhang N, Xing J, Cui M, Yang H, Yao Z, et al: Predictive value of preoperative detection of CEA and CA199 for prognosis in patients with stage II-III colorectal cancer. Zhonghua Wei Chang Wai Ke Za Zhi 18: 914-919, 2015 (In Chinese).

25. Tsigelny IF, Kouznetsova VL, Pingle SC and Kesari S: bHLH Transcription factors inhibitors for cancer therapy: General features for in silico drug design. Curr Med Chem 21: 3227-3243, 2014.

26. Zhu R, Yang Y, Tian Y, Bai J, Zhang X, Li X, Peng Z, He Y, Chen L, Pan Q, et al: Ascl2 knockdown results in tumor growth arrest by miRNA-302b-related inhibition of colon cancer progenitor cells. PLoS One 7: e32170, 2012.

27. Patel D and Chaudhary J: Increased expression of bHLH transcription factor E2A (TCF3) in prostate cancer promotes proliferation and confers resistance to doxorubicin induced apoptosis. Biochem Biophys Res Commun 422: 146-151, 2012.

28. Axelson H: The Notch signaling cascade in neuroblastoma: Role of the basic helix-loop-helix proteins HASH-1 and HES-1. Cancer Lett 204: 171-178, 2004.

29. Sang L, Roberts JM and Coller HA: Hijacking HES1: How tumors co-opt the anti-differentiation strategies of quiescent cells. Trends Mol Med 16: 17-26, 2010. 